and scalars, the book opens with two chapters dealing with Newtonian force fields in two and three dimensions and the corresponding potential functions in that order. This, in itself, seems an unfortunate beginning, for in most of the elementary problems in mathematical physics the force fields are calculated after the potential has been derived. It would seem to be much more reasonable to define the potential as a line-integral, pointing out necessary and sufficient conditions for its existence, and to deduce from it the vector equation $\mathbf{F}=-\nabla U$; and then proceeding to show at once that the Newtonian inverse square law of attraction is derivable from the potential $U=1 / r$, and finally building up the more complicated functions (for example, those due to doublets, surface and volume distributions, and so on) by the ordinary processes of integration and summation.

Chapter 3 discusses the so-called integral theorems : namely, the theorems of Gauss and Stokes and their various corollaries. It is in this chapter that the authors really fail to give their vector analysis a free hand. It is true that the complete proofs of these theorems present formidable topological difficulties; but as these have been laid aside, in any event, as being outside the scope of such a work as this, there can be no excuse for not presenting simple and elegant proofs along formal lines. Furthermore, the theorems of the equivalent layer (or Green's formulæ) follow much more obviously by vector methods than by the (admittedly equivalent) Cartesian methods used in the text.

In the fourth chapter, the ideas of expansion of potential functions in series of spherical harmonics are treated. Much of the convergence theory could, one feels, be cut out with advantage, as it could be supplied by any competent reader, if so desired. In addition, the more elaborate properties of Legendre's polynomials and the associated functions can only be dealt with, satisfactorily, by complex function theory; and one is left with the distasteful impression that, in certain cases, it is only by luck, rather than judgment, that the correct results are obtained. by the cruder arguments involving real variables only.

From Chapter 5 onwards, the book becomes infinitely more interesting, and it is in this and the remaining six chapters that the authors have provided a most valuable contribution to mathematical literature. Chapter 5 gives an account of the behaviour of the potential at points occupied by mass and is sufficiently accurate and comprehensive for most ordinary students. A short chapter on the relations of the two-dimensional theory to that of the functions of a complex variable is followed by a very lucid discussion of the boundary value problems of Dirichlet and Neumann, and a proof of the uniqueness of their solution, if it is assumed to exist.

The problems in the case of the circle and the sphere are solved explicitly in Chapters 8 and 9 , by means of Poisson's integral ; and certain results from the theory of Fourier series, which arise from these, are provided (with proofs where these can be easily supplied). The theory of Green's function is also most adequately dealt with and the existence of this function is shown to be equivalent to that of the mapping function in the fundamental theorem of Riemann, dealing with the conformal representation of any simply connected region with more than one boundary point on the interior of the unit circle.

In the last two chapters the existence of the solution of both the boundary-value problems (pre- viously quoted) is shown to be equivalent to the existence of a solution of the various types of Fredholm's integral-equation, of which an excellent account is given in Chapter 10 .

In spite of having made so much adverse criticism, I think there is no doubt that this book will prove to be of great value as a work of reference. It has been provided with a very detailed table of contents and quite a useful index.

J. H. Pearce.

\section{OPERATIONAL CALCULUS}

Heaviside's Operation Calculus Made Easy

By Dr. T. H. Turney. Pp. vii + 96. (London: Chap. man and Hall, 1944.) 10s. 6d. net.

The Simple Calculation of Electrical Transients

An Elementary Treatment of Transient Problems in Linear Electrical Circuits, by Heaviside's Operational Method. By G. W. Carter. Pp. viii + 120. (Cambridge: At the University Press, 1944.) 8s. $6 d$. net.

CAN any subject be made easy? Did Silvanus C Thompson's "Calculus Made Easy" save a single headache in the younger generation when it tried to master the mathematical tools of its elders? The first of the above books ought to be one which has been long awaited, namely, a graduated introduction to the powerful tool devised by Heaviside. The colloquial style does not, however, inspire confidence ; and the early analogies, for example, that of a battery and condenser, and a general disregard of physical principles in favour of simplifying or grouping mathematical representations, are apt to lead an innocent reader astray. Just interchanging voltages and currents in formulæ simply will not do, even if this bright idea appears to help in simple applications. If the author's deduction (p. 12) that non-proportionality of current and flux in a laminated inductance leads to the idea that "the term inductance has little meaning in any case" were really true, most electrical engineers would be profoundly grateful. Then in two short paragraphs, which seem to have slipped forward, we are suddenly confronted with the properties of $p$. We soon come, however, to $p$ and $j$, Helmholtz's circuit law, and Heaviside's unit function. Through partial fractions we are led to the application of Heaviside's expansion theorem to transmission lines, and to the development of the treatment of impulses. Dr. Turney finally compares Fourier and Heaviside integrals. The author is always on thin ice; although we do not expect rigour in an introduction to a difficult subject, at least we expect discipline. Mathematicians will certainly not commend this book.

The second book has a more restrictive title, although covering a similar section of the subject. The author is on much surer ground, needs no flourishes of style, comes much more quickly to the point at issue, holds on to physical aspects of his problems, and states quite clearly his assumptions of knowledge in the reader; his high-grade examples are closely woven into the text. The student is apt to be diffident in the usage of Heaviside's calculus, except in its simplest applications, in which he often knows the answer from more protracted but less intuitive methods; the present book should through its exercises give students that confidence which is the educational stimulus to go further. Perhaps this is the text-book we have been waiting for.

L. E. C. Hughes. 